\title{
Role of anxiety in the pathophysiology of irritable bowel syndrome: importance of the amygdala
}

\author{
Brent Myers ${ }^{1,2}$ and Beverley Greenwood-Van Meerveld ${ }^{1,2,3 *}$ \\ Veterans Affairs Medical Center, Oklahoma City, OK, USA \\ 2 Oklahoma Center for Neuroscience, University of Oklahoma Health Sciences Center, Oklahoma City, OK, USA \\ ${ }^{3}$ Department of Physiology, University of Oklahoma Health Sciences Center, Oklahoma City, OK, USA
}

\section{Edited by:}

Gary M. Mawe, University of Vermont, Burlington, VT, USA

\section{Reviewed by:}

Timothy Dinan, University College Cork, Cork, Ireland

Michael D. Crowell, Mayo Clinic,

Scottsdale, AZ, USA

\section{*Correspondence:}

Beverley Greenwood-Van Meerveld Veterans Affairs Medical Center,

Research Administration Rm. 151, 921 NE 13th Street, Oklahoma City, OK 73104, USA.

e-mail:Beverley-Greenwood@ ouhsc.edu
A common characteristic of irritable bowel syndrome (IBS) is that symptoms, including abdominal pain and abnormal bowel habits, are often triggered or exacerbated during periods of stress and anxiety. However, the impact of anxiety and affective disorders on the gastrointestinal (GI) tract is poorly understood and may in part explain the lack of effective therapeutic approaches to treat IBS. The amygdala is an important structure for regulating anxiety with the central nucleus of the amygdala facilitating the activation of the hypothalamic-pituitary-adrenal axis and the autonomic nervous system in response to stress. Moreover, chronic stress enhances function of the amygdala and promotes neural plasticity throughout the amygdaloid complex. This review outlines the latest findings obtained from human studies and animal models related to the role of the emotional brain in the regulation of enteric function, specifically how increasing the gain of the amygdala to induce anxiety-like behavior using corticosterone or chronic stress increases responsiveness to both visceral and somatic stimuli in rodents. A focus of the review is the relative importance of mineralocorticoid receptor and glucocorticoid receptor-mediated mechanisms within the amygdala in the regulation of anxiety and nociceptive behaviors that are characteristic features of IBS. This review also discusses several outstanding questions important for future research on the role of the amygdala in the generation of abnormal GI function that may lead to potential targets for new therapies to treat functional bowel disorders such as IBS.

Keywords: irritable bowel syndrome, visceral hypersensitivity, anxiety, amygdala, corticosterone, hypothalamic-pituitaryadrenal axis

\section{INTRODUCTION}

Irritable bowel syndrome (IBS) is a common gastrointestinal (GI) disorder characterized by abnormal bowel patterns and abdominal pain that affects $10-15 \%$ of North Americans (Talley et al., 1992). A prominent feature of IBS symptomatology is altered visceral perception exhibited by hypersensitivity of the colon to luminal distension (Ritchie, 1973; Whitehead et al., 1980). Although the direct cost of IBS is approximately $\$ 1.7$ billion annually, the etiology of the illness is still unknown and no FDA-approved therapies are available to patients (Hulisz, 2004). In this review we discuss the relationship between IBS and anxiety with a special emphasis on the importance of the amygdala as a site integrating emotional behavior and visceral pain processing. Although other brain sites including the cingulate, prefrontal, and insular cortices have been implicated in the regulation of both affective and visceral functions, the vast

Abbreviations: ACTH, adrenocorticotropic hormone; BDNF, brain-derived neurotropic factor; CaMKII, calcium-calmodulin-dependent protein kinase II; CBF, cerebral blood flow; CCK, cholecystokinin; CeA, central nucleus of the amygdala; CNS, central nervous system; CORT, corticosterone; CRD, colorectal distension; CRF, corticotropin releasing factor; ERK, extracellular signal-regulated kinase; fMRI, functional magnetic resonance imaging; GI, gastrointestinal; GR, glucocorticoid receptor; HPA, hypothalamic-pituitary-adrenal; IBS, irritable bowel syndrome; LC, locus coeruleus; MR, mineralocorticoid receptor; NGF, nerve growth factor; OVX, ovariectomized; PAG, periaqueductal gray; PET, positron emission tomography; PKA, protein kinase A; 5-HT, serotonin. connectivity of the amygdala uniquely positions the complex to modulate the function of theses cortical structures and serve as an integrator of emotional and enteric physiology (Bonaz et al., 2002; McDonald et al., 1996; Naliboff et al., 2006; Shi and Cassell, 1998; Wilder-Smith et al., 2004). As we discuss the role of the amygdala in the regulation of visceral pain, we will also provide evidence for a convergence of visceral and somatic sensation because of the significant proportion of IBS patients with co-morbid somatic disorders such as fibromyalgia (Chang et al., 2000; Riedl et al., 2008; Verne et al., 2001). Recent advances in our understanding of altered central nervous system (CNS) activity in IBS patients have emerged from human brain imaging and we will briefly outline the major findings from these studies along with results from small animal imaging to support the hypothesis of amygdala hyperactivity in IBS patients. An additional characteristic of IBS is the marked female predominance of the disorder and we will summarize the sex differences in central processing of visceral stimuli and the lessons learned from animal models on the role of ovarian hormones in the regulation of anxious behavior, visceral sensitivity, and somatic pain. A significant portion of this review will be dedicated to the effects of amygdala manipulation on anxiety, visceral sensation, and somatic thresholds with specific sections devoted to the actions of stress hormones such as corticosteroids (CORT) and corticotropin releasing factor (CRF). We also address other important messengers 
regulating amygdala function including protein kinase A (PKA), extracellular signal-regulated kinase (ERK), calcium-calmodulindependent protein kinase II (CaMKII) and neurotropic factors that may represent targets for novel therapeutic approaches to the treatment of IBS.

\section{ANXIETY AND IBS: WHAT IS THE CONNECTION?}

IBS patients report more frequent stressors and greater impact of stressful events on daily life than controls; additionally, IBS symptomatology is often exacerbated during periods of stress and there is a statistically significant relationship between stress, bowel symptoms, illness-related absenteeism, and medical clinic visits (Blanchard et al., 2008; Whitehead et al., 1990, 1992). Levels of chronic life stress can also predict clinical outcome in IBS patients which can be demonstrated by the temporal relationship between life stress and the subsequent intensity of bowel symptoms (Bennett et al., 1998). Furthermore, a statistical correlation among mean daily stress, indices of anxiety and depression, and GI symptoms including abdominal pain, bloating, and altered bowel patterns suggests that altered activity in stress-regulating pathways may be an important component of IBS symptomatology (Hertig et al., 2007). Recent clinical studies have implicated hypothalamic-pituitary-adrenal (HPA) axis dysregulation in the pathophysiology of IBS by demonstrating that patients possess elevated cortisol levels both at baseline and in response to stress (Chang et al., 2009; Dinan et al., 2006). Additionally, the CRF stimulation test has been used to demonstrate exaggerated adrenocorticotropic hormone (ACTH), cortisol, and colonic motility responses in IBS patients compared to healthy controls (Dinan et al., 2006; Fukudo et al., 1998). This stress hyper-responsiveness may be responsible for the increased level of psychopathology in IBS patients compared to patients with other GI disorders such as inflammatory bowel disease (Blanchard et al., 1990). The diagnosis rate of psychiatric disorders in treatment-seeking IBS patients ranges from 54 to $94 \%$ and anxiety disorders are the most common psychiatric co-morbidities with up to $61 \%$ of treatment-seeking IBS patients possessing a DSM-IV-diagnosed anxiety disorder (Drossman et al., 1999; Whitehead et al., 2002). Clinical studies consistently report an association between anxiety and IBS with panic, generalized anxiety, and post-traumatic stress representing the most prevalent anxiety disorders in IBS patients (Blanchard et al., 1990; Jones et al., 2006; Lydiard, 2001). Although there is an argument that these findings may be a result of selection bias because these studies only examine treatmentseeking patients who may be more prone to medical complaints in general, a community-based survey found not only that rates of clinically diagnosed anxiety disorders were significantly higher in IBS patients than the general population but also that the rates for nontreatment-seeking patients were similar to treatment-seekers (Lydiard and Falsetti, 1999). IBS patients also show increased fear-potentiated acoustic startle responses compared with controls, suggesting central hyper-excitability and increased vigilance (Naliboff et al., 2008). Although these findings do not eliminate the possibility that treatment-seekers possess atypical illness-related attitudes and/or hypochondriacal personalities, they offer strong support for the hypothesis that anxiety and IBS share common biological factors.
Our group has demonstrated that, in rodents, there is a link between the central pathways mediating stress and anxiety and the mechanisms regulating GI sensitivity (Greenwood-Van Meerveld et al., 2001). A key component of this link is the amygdala, specifically the central nucleus of the amygdala (CeA). The role of the amygdala in the regulation of emotional behavior is well established; however, recent work has shown that this limbic structure integrates emotional and sensory information and is a vital site for the expression of fear and anxiety (Davis, 1992, 1997; Phelps and LeDoux, 2005; Schulkin et al., 1994; Weiskrantz, 1956). Additionally, the amygdala is involved in the learning, consolidation, and acquisition of emotional memories and has an essential role in classically conditioned responses (Davis, 1997; LeDoux, 2000). These functions are closely related to the role of the amygdala in the generation of fear and may be important for IBS as studies in rodents have demonstrated that colonic sensitivity and motility are increased following fear conditioning (Gue et al., 1991; Tyler et al., 2007). There are approximately 13 nuclei of the amygdala that are anatomically and physiologically heterogeneous but these structures can be grouped into three complexes based on functional interactions (Sah et al., 2003). These are the frontotemporal (basal, lateral, and accessory basal nuclei), autonomic (central and medial nuclei), and olfactory groups (main and accessory olfactory systems) (Swanson, 2000; Swanson and Petrovich, 1998). The CeA facilitates both the autonomic and endocrine responses to stress and stimulation of the CeA produces activity consistent with heightened anxiety such as enhanced startle, freezing behavior, and increased plasma CORT (Applegate et al., 1983; Dunn and Whitener, 1986; Rosen and Davis, 1988). Conversely, lesions of the CeA decrease anxiety-like behaviors associated with conditioned fear, novel environments, and social stress (Burns et al., 1996; Roozendaal et al., 1990). CeA lesions also reduce baseline CRF expression in the hypothalamus and stress-induced release of ACTH and CORT (Beaulieu et al., 1986; Prewitt and Herman, 1994). Anatomical studies indicate that the CeA provides major output to autonomic regions that mediate not only fear and anxiety-related behaviors but also enteric processes such as gastric emptying and colonic motility (LeDoux et al., 1988; Lyubashina, 2004; Swanson and Petrovich, 1998). A summary of the anatomical connectivity of the amygdala with projections relevant to the integration of visceral and somatic pain, motor activity of the GI tract, as well as stress and anxiety is displayed in Figure 1. Although the role of the amygdala in the regulation of GI motor function is an understudied area, electrical stimulation of the CeA can inhibit gastric motility through direct projections from the CeA to the dorsal motor nucleus of the vagus and nucleus of the solitary tract (Krettek and Price, 1978; Lyubashina, 2004; Schwaber et al., 1982). In addition, Figure 2 illustrates the projections from the CeA to the locus coeruleus (LC) and Barrington's nucleus that modify distal colonic motility through manipulation of the sacral parasympathetic nucleus (Sakanaka et al., 1986; Valentino et al., 1999). The network of projections from the CeA to the periaqueductal gray (PAG), LC, raphe nuclei, and parabrachial nucleus participates in ascending arousal and affective systems as well as the descending modulation of nociceptive afferents and represents a mechanism for altered visceral and somatic sensation (Davis, 1997; Jones et al., 2006; Sah et al., 2003). Given that episodes of anxiety and stress are linked to the symptomatology of IBS, we hypothesize 


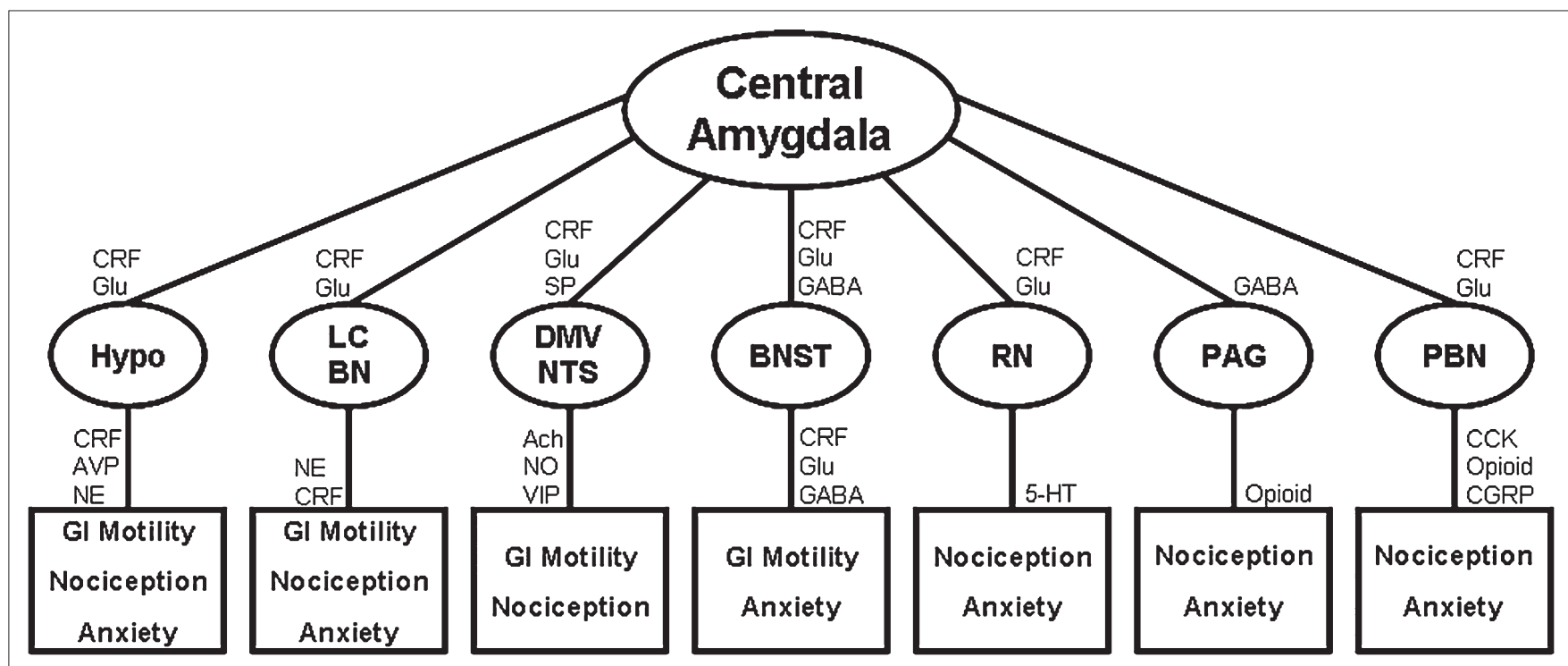

FIGURE 1 | Mechanisms for amygdala-mediated integration of abdominal and cutaneous pain, GI motility, and anxiety. All projections are reciprocal and connectivity is simplified for clarity. Ach, acetylcholine; AVP, arginine vasopressin; BN, Barrington's nucleus; BNST, bed nucleus of the stria terminalis; CCK, cholecystokinin; CGRP, calcitonin gene-related peptide; CRF, corticotropin releasing factor; DMV, dorsal motor nucleus of the vagus; Glu, glutamate; Hypo, hypothalamus; LC, locus coeruleus; NE, norepinephrine; NO, nitric oxide; NTS, nucleus of the solitary tract; PAG, periaqueductal gray; PBN, parabrachial nucleus; $R N$, raphe nuclei; SP, substance $P$; VIP, vasoactive intestinal peptide; 5-HT, serotonin (Choi et al., 2007; Davis, 1997; Gray and Magnuson, 1987; Hermanson et al., 1998; Jones et al., 2006; Niedringhaus et al., 2008; Sah et al., 2003; Sakanaka et al., 1986).

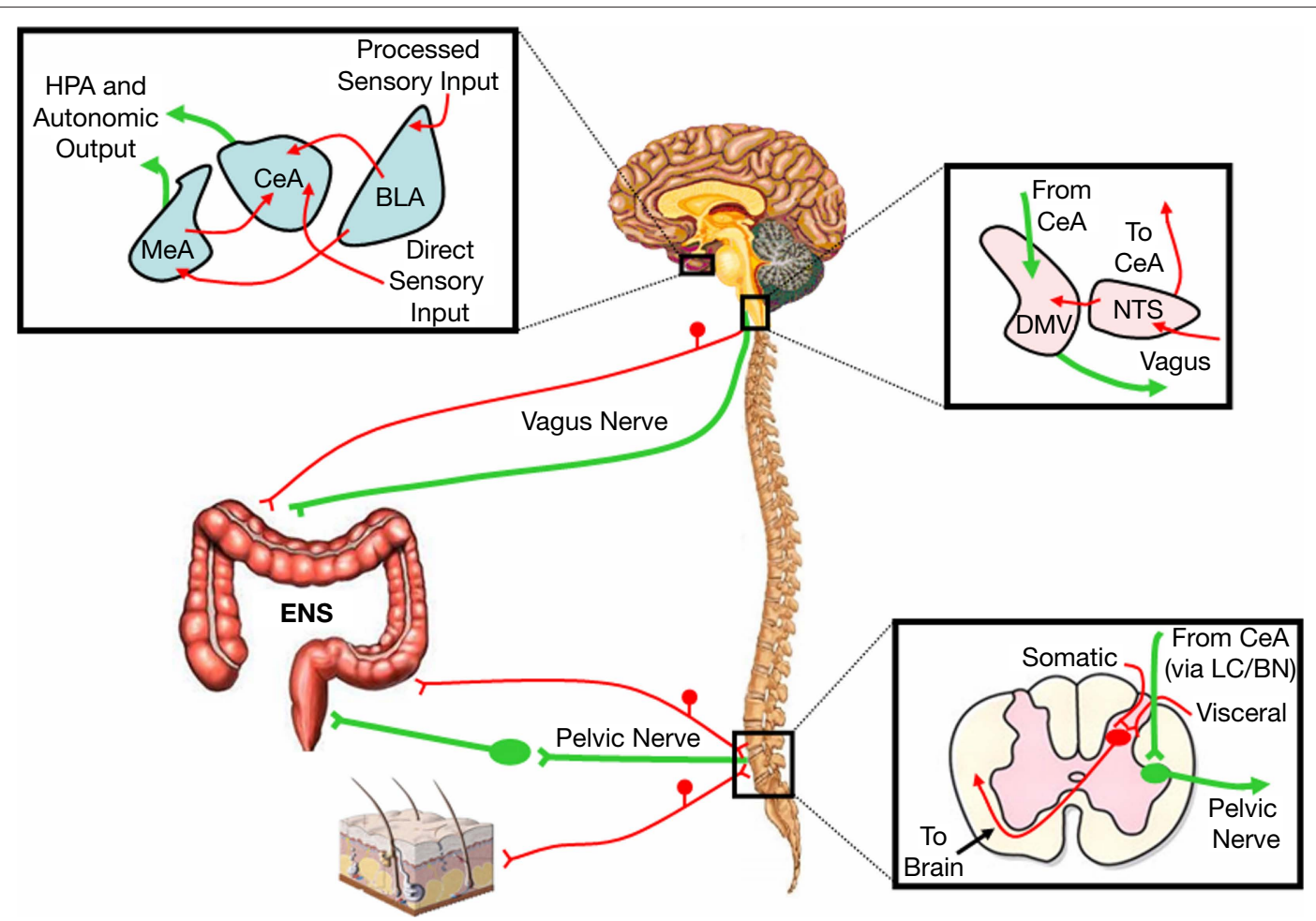

FIGURE 2 | Hypothesized pathways representing the primary amygdalaperipheral interactions. Sensory-motor interactions between the amygdala and proximal colon are mediated by the vagus nerve and the nuclei of the dorsal vagal complex. The amygdala also influences distal colonic motility through the pelvic nerve via the LC/BN complex while convergence of visceral and somatic afferents occurs in the lumbosacral spinal cord. Red represents afferent pathways and green depicts efferent connections. BLA, basolateral nucleus of the amygdala; BN, Barrington's nucleus; $\mathrm{CeA}$, central nucleus of the amygdala; DMV, dorsal motor nucleus of the vagus; ENS, enteric nervous system; HPA, hypothalamic-pituitary-adrenal; LC, locus coeruleus; MeA, medial nucleus of the amygdala; NTS, nucleus of the solitary tract. 
that a connection exists between the amygdala and enteric responses to behavioral situations.

\section{IMAGING THE NEURAL CIRCUITRY OF AMYGDALA HYPERACTIVITY}

In recent years, imaging studies using both functional magnetic resonance imaging (fMRI) and positron emission tomography (PET) have demonstrated that, compared to healthy controls, IBS patients display altered central processing of visceral sensory information. In healthy subjects, visceral stimulation consistently activates the insular cortex, an important area for perception of internal body states, and the anterior portion of the cingulate cortex, a region that mediates affective-sensory and motivational processes (Derbyshire, 2003). These studies have also demonstrated activation in other areas including the primary sensory and prefrontal cortices (Derbyshire, 2003). IBS patients show activation of many of the same structures but also show substantial activation of the hypothalamus and amygdala as well as decreased activity of the antinociceptive PAG (Naliboff et al., 2001). More recent investigations employing rectosigmoid balloon distension in IBS patients have shown activity in a network of brain structures involved in both sensory-discriminative functions as well as emotional-motivational processes. Specifically, areas regulating affective and sensory processes including the amygdala, insula, cingulate, and prefrontal cortex show greater activation in IBS patients than controls (Naliboff et al., 2003; Wilder-Smith et al., 2004). Additionally, neuroimaging studies in subjects with mood disorders have shown that resting cerebral blood flow $(\mathrm{CBF})$ and glucose metabolism are abnormally elevated in the amygdala and elevations in amygdala CBF persist for an abnormally long time in response to a stressor (Drevets, 2003). Although fMRI is a powerful tool for noninvasive imaging of brain function, very little research has used the technique to examine central processing in animal models of visceral hypersensitivity. However, in a study performed in anesthetized adult rats, fMRI was used to detect brain areas activated by colorectal distension (CRD) (Lazovic et al., 2005). Additionally, fMRI activation was compared with postmortem histological analysis of the immediate early gene product $c$-fos to establish whether fMRI results are comparable to $c$-fos expression. The CeA displayed a significant number of $c$-fos positive cells in response to visceral stimulation and all rats undergoing fMRI exhibited activation of the amygdaloid complex in response to CRD. This study also demonstrated that, although $c$-fos had greater resolution for identifying neuronal activation in specific subnuclei, fMRI was a more sensitive and selective method for comparing animals undergoing CRD with controls. In recent work, these observations have been advanced by showing that, in anesthetized rats, there are significant differences in brain activation in response to CRD following modulation of amygdala function (Johnson et al., 2007). This was accomplished by investigating whole brain fMRI in anesthetized rats to identify brain regions activated by visceral stimulation following exposure of the amygdala to high levels of CORT. These experiments provided evidence that elevated amygdaloid CORT significantly increases brain activation in response to visceral stimulation compared to controls, suggesting a stress-induced afferent neuronal hypersensitivity. The information obtained in this study provided pivotal data supporting the hypothesis that the amygdala is important for the sensitization of a neuronal sensory circuit and these results complement studies in conscious rats in which CORT was stereotaxically administered onto the amygdala and found to induce anxiety and a heightened visceromotor behavioral response to CRD (Myers and GreenwoodVan Meerveld, 2007). However, one limitation of data from these animal imaging studies is that the animals must be anesthetized while, in clinical studies, IBS patients are awake. To address this issue, others have utilized CRD in awake, nonrestrained rodents injected with a radioactive tracer for quantifying regional CBF (Wang et al., 2008). Although the autoradiographic reconstruction of $\mathrm{CBF}$ occurred postmortem, the analysis yielded interesting results including increases in $\mathrm{CBF}$ in the amygdala, insula, and anterior cingulate cortex. Overall, brain imaging studies from both humans and rodents suggest that hyperactivity of the amygdala plays an important role in the altered central processing of visceral information seen in IBS patients.

\section{THE ROLE OF GENDER}

Irritable bowel syndrome is one of the most common disorders outside of gynecological diseases that influence women's work productivity due to frequent and potentially disabling symptoms. Studies have demonstrated that approximately two-thirds of individuals with IBS are female, with an estimated prevalence in women ranging from 14-24\% (Drossman et al., 1997). Similarly, anxiety disorders are twice as prevalent in females compared to males providing further evidence for common biological factors in the pathophysiology of these disorders (Seeman, 1997). A potential explanation for the female predominance of IBS is an alteration of CNS processing of visceral information. Studies using PET imaging suggest that there are gender differences in regional brain responses to rectal pressure in IBS patients; specifically, women with IBS show greater activation of brain regions associated with affective responses including the amygdala and cingulate cortex when compared to men with IBS (Berman et al., 2000; Naliboff et al., 2003). Another factor involved in the female predominance of IBS relates to the observation that IBS symptomatology fluctuates with changes in the female reproductive cycle suggesting a role for ovarian hormones in mediating symptom severity (Heitkemper et al., 2003). Specifically, IBS symptomatology worsens following the peak of estradiol and progesterone in the luteal phase and this exacerbation continues into early menses (Crowell et al., 1994; Heitkemper et al., 1995). Additionally, concentrations of cortisol, epinephrine, and norepinephrine in the urine of female IBS patients are significantly elevated throughout the luteal phase compared to healthy controls (Heitkemper et al., 1996). There is an extensive body of literature supporting multiple interactions between ovarian hormones, reproductive states, and the HPA axis which may represent mechanisms for ovarian hormone-induced increases in IBS symptomatology. For instance, female rats typically secrete higher basal levels of CORT and show greater ACTH and CORT responses to stress. This sex difference is abolished by ovariectomy (OVX) and reinstated by estradiol administration (Viau and Meaney, 1991). Estrogens may mediate these effects by directly interacting with the CRF gene to promote CRF expression and HPA activity in addition to interfering with CORT-dependent negative feedback leading to increased HPA function both at rest and following a stressor (Vamvakopoulos and Chrousos, 1993; Weiser and Handa, 
2009). In animal studies of GI function, estrous cycle variations in visceral pain thresholds have been observed that mirror those seen in human subjects. Specifically, proestrus, a phase characterized by elevated estradiol and progesterone, is associated with colonic hypersensitivity; furthermore, OVX females display decreased visceral sensitivity and afferent excitability compared to intact females (Ji et al., 2003, 2008; Sapsed-Byrne et al., 1996). Research focused on the ability of exogenous estrogens to alter nociceptive and anxiety-like behaviors has led to surprisingly contradictory results; however, there is evidence suggesting that estradiol modulates anxiety and fear-related behaviors, visceral sensitivity, and somatic pain (Frye and Walf, 2004; Ji et al., 2003; Kayser et al., 1996; Morgan and Pfaff, 2001). While the effects of progesterone are somewhat less clear, there is evidence to support increases in indices of anxiety-like behavior and visceral sensitivity as well as altered somatic sensitivity following systemic administration (Frye and Walf, 2004; Gue, 2000; Gulinello and Smith, 2003). Although the specific brain sites mediating the effects of ovarian hormones on anxiety and visceral sensitivity are not well defined, a growing body of evidence suggests that the amygdala plays a critical role as a sexually dimorphic region that expresses receptors for ovarian steroids (Frye and Walf, 2004; Greco et al., 2001; Milner et al., 2008; Parsons et al., 1982; Rainbow et al., 1982). For instance, a recent fMRI study of women in the follicular phase utilized oral administration of progesterone to mimic hormone levels present during the luteal phase and found an increase in CNS activity that was highly specific to the amygdala (Van Wingen et al., 2008). Clearly more research is required to fully understand the effects of ovarian hormones on IBS symptomatology but the available evidence suggests that estradiol and progesterone are important modulators of affective and nociceptive behaviors.

\section{NEUROENDOCRINE MODULATION OF THE AMYGDALA: EFFECTS ON ANXIETY, VISCERAL SENSITIVITY, AND SOMATIC PAIN CORTICOSTEROIDS}

Similar to other structures in the CNS that modulate HPA activity, neurons within the amygdala express corticosteroid receptors with the highest density found in the CeA (Sapolsky et al., 1983). Corticosteroid hormones released from the adrenal cortex act through two receptor subtypes, glucocorticoid receptors (GR), and mineralocorticoid receptors (MR), which differ in their distribution and pharmacological properties (De Kloet and Reul, 1987). Specifically, effects attributed to MR activation include maintenance of blood pressure and ion balance in target tissues such as the kidney, colon, and salivary glands (Marver, 1984), whereas the effects of GR activation include regulation of carbohydrate and amino acid metabolism and modulation of inflammatory responses (Munck and Naray-Fejes-Toth, 1992). Receptor binding studies have suggested that CORT acts through MR during basal levels of HPA axis activity, whereas GR regulates CORT activity during more stressful conditions (De Kloet et al., 1998; Pace and Spencer, 2005). Under normal neuroendocrine conditions, the effect of elevated CORT in the brain and periphery is largely inhibitory as a component of the negative feedback response to stress. Importantly, IBS patients display not only elevated plasma CORT (Chang et al., 2009; Dinan et al., 2006) but also abnormal amygdala activity (Naliboff et al., 2003; Wilder-Smith et al., 2004) and this altered balance in stress modulation induced by amygdala hyperactivity may represent an essential aspect of visceral hypersensitivity. Therefore, it is the aberrant amygdala activity in the presence of elevated CORT that we consider an important component of IBS symptomatology. Our findings that stereotaxic delivery of CORT to the amygdala enhances anxiety-like behavior coupled with heightened visceral sensitivity and lowered somatic pain thresholds reinforce a direct interaction between corticosteroids and the amygdala (Myers and Greenwood-Van Meerveld, 2007; Myers et al., 2007). In these studies the hippocampus and caudate putamen were also targeted with CORT micropellets and these placement controls demonstrated that the CORT-induced anxiety and viscero-somatic hypersensitivity were specific to the amygdala. Although corticosteroids are known to affect inflammatory and immune processes, an additional series of experiments confirmed that amygdaloid CORT implants and the subsequent increase in peripheral CORT do not affect the morphology or immune response of the colonic mucosa. After establishing that the effects of CORT implants on anxiety and pain sensitivity were specific to the amygdala and did not involve changes in the integrity of the colon, we sought to investigate the relative contribution of the two types of corticosteroid receptors using selective antagonists to GR or MR. Combining the GR antagonist mifepristone with CORT revealed a significant reduction in anxiety and colonic sensitivity while spironolactone, an MR antagonist, combined with CORT also induced a significant inhibition of anxiety and colonic sensitivity. Therefore, at the level of the amygdala, both GR and MR play critical roles in the regulation of IBS symptomatology.

\section{CORTICOTROPIN RELEASING FACTOR}

Corticotropin releasing factor is a key neuropeptide that has been implicated in the neuroendocrine, autonomic, immune and behavioral responses to stress and acts through two main receptor subtypes, type $1\left(\mathrm{CRF}_{1}\right)$ and type $2\left(\mathrm{CRF}_{2}\right)$ (De Souza et al., 1984). Pharmacological studies of CRF action in the CNS and periphery have demonstrated the capacity of this neuropeptide to exert major effects on enteric function including gastric emptying, colonic motility, and mucosal permeability; however, this area will not be the focus of this review due to the extensive literature describing the effects of CRF on GI function (Tache and Bonaz, 2007; Tache and Brunnhuber, 2008; Tache and Perdue, 2004; Tache et al., 1993). While endogenous CRF has been shown to play a role in mediating stress-induced changes in GI motility and colonic sensitivity, accumulating evidence from animal models suggests that CRF in the amygdala may play a role in IBS symptomatology (Gue et al., 1991). For example, both emotional stress (contextual fear conditioning) and central administration of CRF increase colonic motility and these effects are inhibited by direct injection of a cholecystokinin (CCK) antagonist into the CeA. Interestingly, the inhibitory effects of the CCK antagonist are abolished by lesioning the CeA suggesting that the amygdala is an important site for mediating the effects of CRF on enteric function (Gue et al., 1994). Central administration of CRF also elicits anxiety and fear-related behaviors while direct injection of CRF antagonists into the CeA blocks these effects (Swiergiel et al., 1993). Furthermore, Wistar-Kyoto rats have been shown to have higher levels of anxiety and visceral sensitivity than 
other rat strains and these findings correlate with increased expression of CRF in the CeA (Gunter et al., 2000; Shepard and Myers, 2008). Modulation of the amygdala with CORT also upregulates CRF mRNA expression in the CeA and the effects of elevated amygdaloid CORT on anxiety-like behavior and visceral hypersensitivity are reversed by antalarmin, $\mathrm{CRF}_{1}$ receptor antagonist (Myers et al., 2005; Shepard et al., 2000). Additional evidence for the importance of amygdaloid CRF in the regulation of enteric physiology comes from a study showing that trinitrobenzenesulfonic acid-induced colitis leads to increased CRF mRNA expression in the CeA and paraventricular nucleus of the hypothalamus (Greenwood-Van Meerveld et al., 2006) demonstrating that acute GI inflammation activates the CeA to induce the expression of CRF. The role of amygdaloid CRF in somatic pain processing and pain-related anxiety has been well characterized in the kaolin/carrageenan model of arthritis. In rats with arthritis-like pain in the knee, a selective $\mathrm{CRF}_{1}$ receptor antagonist injected directly into the CeA inhibits anxiety and nociceptive reflexes induced by mechanical stimulation of the knee (Ji et al., 2007). Additional studies focused on the specific roles of $\mathrm{CRF}_{1}$ and $\mathrm{CRF}_{2}$ receptors in the amygdala have shown that $\mathrm{CRF}_{1}$, by coupling to the PKA intracellular signaling system, is pronociceptive while $\mathrm{CRF}_{2}$ is antinociceptive but only active under basal conditions (Ji and Neugebauer, 2008). Since CRF is integral in mediating the changes that accompany stress and anxiety, this peptide may provide a common link in the integration of behavioral and visceral responses to stress.

\section{QUESTIONS FOR FUTURE RESEARCH AND THE DEVELOPMENT OF NOVEL THERAPEUTIC TARGETS}

Although our knowledge of amygdala-mediated mechanisms that regulate IBS symptomatology has greatly increased, there are several outstanding questions that could direct future research toward finding effective treatments for functional GI disorders. With the knowledge that corticosteroid receptors and CRF in the amygdala play prominent roles, determining the specific cellular mechanisms regulating their function is essential. Both MR and GR act as transcription factors regulating the expression of many target genes. While some of these genes and their protein and enzyme products have been discovered including $\mathrm{CRF}_{1}$, monoamine oxidase $\mathrm{A}$, serotonin (5-HT) receptor $1 \mathrm{~A}$, and GABA receptor $\mathrm{A}$, there has been no systematic analysis of MR and GR responsive genes in the amygdala or the role they may play in the regulation of anxiety and visceral hypersensitivity (Morsink et al., 2006). Identifying these target genes and the specific isoforms of receptors, enzymes, and peptides regulated by corticosteroids could provide promising therapeutic targets. For example, the interaction of CORT and 5-HT in the amygdala represents a potentially important aspect of anxiety and chronic pain yet has received little attention. Altered intestinal 5-HT signaling in IBS has been documented as has the ability of CORT to regulate 5-HT receptor expression and binding in the hippocampus and extracellular 5-HT concentrations in the hypothalamus; conversely, the effects of CORT on 5-HT in the amygdala are much less clear (Chalmers et al., 1993; Feng et al., 2009; Mawe et al., 2006). However, preliminary studies suggest that 5-HT has CORT-dependent effects on sensory processing in the lateral amygdala and that behavioral stress increases extracellular 5-HT levels in the amygdala (Rueter and Jacobs, 1996; Stutzmann et al., 1998). The body of knowledge regarding the intracellular signaling of g-protein-coupled CRF receptors is growing with evidence that PKA and ERK are involved in pain-related synaptic plasticity and behavior; however, research is needed to understand the regulation of these receptors as well as their downstream signaling pathways (Fu et al., 2008). For instance, PKA enzymatic activity can be induced by the elevation of cAMP following $\mathrm{CRF}_{1}$ activation; however, the targets of PKA catalysis as well as potential transcriptional interactions between PKA and cAMP response elements in the amygdala related to pain and anxiety are largely unknown. Although ERK is one downstream target of PKA in the regulation of neuronal excitability, ERK intracellular signals can activate many transcriptional factors and protein kinases in addition to mediating interactions between the NMDA receptor and brain-derived neurotropic factor (BDNF) (Hu and Gereau, 2003; Slack et al., 2004). There is also a need for long-term studies to address the amygdaloid mechanisms involved in the development of chronic visceral pain and anxiety. Potential research targets involved in the remodeling associated with chronic visceral hypersensitivity would include the NMDA receptor and downstream CaMKII which have a demonstrated involvement in CRF and stress-related synaptic plasticity leading to long-term changes in amygdala activity and behavior (Rainnie et al., 2004). Intracellular calcium/calmodulin is responsible for the initial activation of CaMKII although the ability of the enzyme to autophosphorylate allows for activity to continue after the initial rise in intracellular calcium. This sustained activity is important for synaptic transmission and plasticity as CaMKII is required for long-term potentiation and experience-dependent neuronal plasticity (Lisman et al., 2002). The role of amygdala hypertrophy is also of special interest in studying the neuroplasticity involved in chronic symptomatology; however, the relative importance of amygdaloid neurotropic factors such as BDNF, nerve growth factor (NGF), and their tyrosine kinase receptors (TrkB and $\operatorname{TrkA}$, respectively) in the regulation of anxiety and visceral hypersensitivity has yet to be determined. Neurotropins generally promote neuronal survival along with the growth and differentiation of new neurons and synapses. Specifically, BDNF appears to be involved in the integration of excitatory and inhibitory neurotransmission and emerging evidence suggests that amygdaloid BDNF can regulate anxiety-like behaviors (Pandey et al., 2006; Slack et al., 2004). NGF is released in response to inflammation and can sensitize primary afferents which may contribute to the development of central sensitization. Interestingly, the majority of visceral afferents express the NGF receptor TrkA and NGF has been shown to regulate pain sensitivity (Di Castro et al., 2006; Hunt and Mantyh, 2001). Although the interactions between neurotropins, their receptors, and other signaling pathways in the amygdala are not well understood, these processes may represent a critical aspect of chronic visceral pain and anxiety.

One cautionary note involves the use of animal models to address these questions as there are several models of stress-related visceral hypersensitivity based largely on the effects of either earlylife trauma or behavioral stressors. The early-life trauma models involve neonatal rats receiving repetitive challenges such as colonic distension, maternal separation, or fear conditioning that lead to visceral hypersensitivity in adulthood while behavioral models in adult rats utilize physical stressors such as restraint 
stress and psychological stressors including water avoidance stress to induce visceral hypersensitivity (Al-Chaer et al., 2000; Gue et al., 1997; Rosztocy et al., 2003; Schwetz et al., 2004; Tyler et al., 2007). However, animal models have important limitations that must be considered when interpreting the results of these studies. First, the nature of the stressors, including whether they are physical/psychological, acute/chronic, or predictable/unpredictable, can have a profound influence not only on the outcome of the investigation but also on the biological processes involved. In many animal models stress responses will habituate to the repeated presentation of a homotypic stressor; therefore, models that do not develop habituation or unpredictable stressors may better mimic the human pathophysiological condition. Second, many of the stress models used to study visceral pain tend to induce exaggerated pain responses or hyperalgesia and, although IBS patients also display hyperalgesia, painful responses to innocuous stimuli or allodynia is a more common clinical complaint. This issue has

\section{REFERENCES}

Al-Chaer, E. D., Kawasaki, M., and Pasricha, P. J. (2000). A new model of chronic visceral hypersensitivity in adult rats induced by colon irritation during postnatal development. Gastroenterology 119, 1276-1285.

Applegate, C. D., Kapp, B. S., Underwood, M. D., and McNall, C. L. (1983). Autonomic and somatomotor effects of amygdala central N. stimulation in awake rabbits. Physiol. Behav. 31, 353-360.

Beaulieu, S., Di Paolo, T., and Barden, N. (1986). Control of ACTH secretion by the central nucleus of the amygdala: implication of the serotoninergic system and its relevance to the glucocorticoid delayed negative feedback mechanism. Neuroendocrinology 44, 247-254.

Bennett, E. J., Tennant, C. C., Piesse, C., Badcock, C. A., and Kellow, J. E. (1998). Level of chronic life stress predicts clinical outcome in irritable bowel syndrome. Gut 43, 256-261.

Berman, S., Munakata, J., Naliboff, B. D., Chang, L., Mandelkern, M., Silverman, D., Kovalik, E., and Mayer, E. (2000). Gender differences in regional brain response to visceral pressure in IBS patients. Eur. J. Pain 4, 157-172.

Blanchard, E. B., Lackner, J. M., Jaccard, J., Rowell, D., Carosella, A. M., Powell, C., Sanders, K., Krasner, S., and Kuhn, E. (2008). The role of stress in symptom exacerbation among IBS patients. J. Psychosom. Res. 64, 119-128.

Blanchard, E. B., Scharff, L., Schwarz, S. P., Suls, J. M., and Barlow, D. H. (1990). The role of anxiety and depression in the irritable bowel syndrome. Behav. Res. Ther. 28, 401-405.

Bonaz, B., Baciu, M., Papillon, E., Bost, R., Gueddah, N., Le Bas, J. F., Fournet, J., and Segebarth, C. (2002). Central processing of rectal pain in patients with IBS: an fMRI study. Am. J. Gastroenterol. 97, 654-661.

Burns, L. H., Annett, L., Kelley, A. E., Everitt, B. J., and Robbins, T.W. (1996). Effects of lesions to amygdala, ventral subiculum, medial prefrontal cortex, and nucleus accumbens on the reaction to novelty: implications for limbic-striatal interactions. Behav. Neurosci. 110, 60-73.

Chalmers, D. T., Kwak, S. P., Mansour, A., Akil, H., and Watson, S. J. (1993). Corticosteroids regulate brain hippocampal 5-HT1A receptor mRNA expression. J. Neurosci. 13, 914-923.

Chang, L., Mayer, E. A., Johnson, T., Fitzgerald, L. Z., and Naliboff, B. (2000). Differences in somatic perception in female patients with irritable bowel syndrome with and without fibromyalgia. Pain 84, 297-307.

Chang, L., Sundaresh, S., Elliott, J., Anton, P. A., Baldi, P., Licudine, A., Mayer, M., Vuong, T., Hirano, M., Naliboff, B. D., Ameen, V.Z., and Mayer, E. A. (2009). Dysregulation of the hypothalamicpituitary-adrenal (HPA) axis in irritable bowel syndrome. Neurogastroenterol. Motil. 21, 149-159.

Choi, D. C., Furay, A. R., Evanson, N. K., Ostrander, M. M., Ulrich-Lai, Y. M., and Herman, J. P. (2007). Bed nucleus of the stria terminalis subregions differentially regulate hypothalamic-pituitary-adrenal axis activity: implications for the integration of limbic inputs. J. Neurosci. 27, 2025-2034.

Crowell, M. D., Dubin, N. H., Robinson, J. C., Cheskin, L. J., Schuster, M. M., Heller, B. R., and Whitehead, W. E. (1994). Functional bowel disorders in women with dysmenorrhea. Am. J. Gastroenterol. 89, 1973-1977.

particular relevance for translational work focused on the development of therapeutics as different neurobiological mechanisms are involved in allodynia and hyperalgesia. Finally, IBS is a complex, multifactoral disorder involving higher cognitive and cortical function as well as complicated interactions between biological, psychological, and sociological variables that no animal model will be able to mimic entirely. Taking these limitations into account, the authors still consider the ascending and descending connectivity of the amygdala to represent important mechanisms for better understanding IBS pathophysiology and possibly improving therapeutic outcomes for patients.

\section{ACKNOWLEDGMENTS}

Beverley Greenwood-Van Meerveld is supported by a Career Scientist Award from the Department of Veterans Affairs and Brent Myers is supported by a Predoctoral Research Fellowship from the Department of Veterans Affairs.

Davis, M. (1992). The role of the amygdala in fear and anxiety. Annu. Rev. Neurosci. 15, 353-375.

Davis, M. (1997). Neurobiology of fear responses: the role of the amygdala. J. Neuropsychiatry Clin. Neurosci. 9, 382-402.

De Kloet, E. R., and Reul, J. M. (1987) Feedback action and tonic influence of corticosteroids on brain function: a concept arising from the heterogeneity of brain receptor systems. Psychoneuroendocrinology 12, 83-105.

De Kloet, E. R., Vreugdenhil, E. Oitzl, M. S., and Joels, M. (1998). Brain corticosteroid receptor balance in health and disease. Endocr. Rev. 19, 269-301.

De Souza, E. B., Perrin, M. H., Insel, T. R. Rivier, J., Vale, W. W., and Kuhar, M. J. (1984). Corticotropin-releasing factor receptors in rat forebrain: autoradiographic identification. Science 224, 1449-1451.

Derbyshire, S. W. (2003). A systematic review of neuroimaging data during visceral stimulation. Am. J. Gastroenterol. 98, 12-20.

Di Castro, A., Drew, L. J., Wood, J. N., and Cesare, P. (2006). Modulation of sensory neuron mechanotransduction by PKC- and nerve growth factordependent pathways. Proc. Natl. Acad. Sci. 103, 4699-4704.

Dinan, T., Quigley, E., Ahmed, S. Scully, P., O’Brien, S., O’Mahony, L. O'Mahony, S., Shanahan, F., and Keeling, P. W. (2006). Hypothalamicpituitary-gut axis dysregulation in irritable bowel syndrome: plasma cytokines as a potential biomarker? Gastroenterology 130, 304-311.

Drevets, W. C. (2003). Neuroimaging abnormalities in the amygdala in mood disorders. Ann. N. Y. Acad. Sci. 985, 420-444.
Drossman,D.A., Creed, F.H., Olden, K. W., Svedlund, J., Toner, B. B., and Whitehead,W.E. (1999). Psychosocial aspects of the functional gastrointestinal disorders. Gut 45, II25-II30.

Drossman, D. A., Whitehead, W. E., and Camilleri, M. (1997). Irritable bowel syndrome: a technical review for practice guideline development. Gastroenterology 112, 2120-2137.

Dunn, J. D., and Whitener, J. (1986). Plasma corticosterone responses to electrical stimulation of the amygdaloid complex: cytoarchitectural specificity. Neuroendocrinology 42 , 211-217.

Feng, N., Telefont, M., Kelly, K. J., Orchinik, M., Forster, G. L., Renner, K. J., and Lowry, C. A. (2009). Local perfusion of corticosterone in the rat medial hypothalamus potentiates d-fenfluramine-induced elevations of extracellular 5-HT concentrations. Horm. Behav. (Epub ahead of print), PMID: 19371745.

Frye, C.A., and Walf,A.A. (2004). Estrogen and/or progesterone administered systemically or to the amygdala can have anxiety-, fear-, and pain-reducing effects in ovariectomized rats. Behav. Neurosci. 118, 306-313.

Fu, Y., Han, J., Ishola, T., Scerbo, M., Adwanikar, H., Ramsey, C., and Neugebauer, V. (2008). PKA and ERK, but not PKC, in the amygdala contribute to pain-related synaptic plasticity and behavior. Mol. Pain 4, 26.

Fukudo, S., Nomura, T., and Hongo, M. (1998). Impact of corticotropinreleasing hormone on gastrointestinal motility and adrenocorticotropic hormone in normal controls and patients with irritable bowel syndrome. Gut42, 845-849.

Gray, T. S., and Magnuson, D. J. (1987). Neuropeptide neuronal efferents from 
the bed nucleus of the stria terminalis and central amygdaloid nucleus to the dorsal vagal complex in the rat. J. Comp. Neurol. 262, 365-374.

Greco, B., Allegretto, E. A., Tetel, M. J., and Blaistein, J.D. (2001). Coexpression of $\mathrm{ER} \beta$ with ER $\alpha$ and progestin receptor proteins in the female rat forebrain: effects of estradiol treatment. Endocrinology 142, 5172-5181.

Greenwood-Van Meerveld, B., Gibson, M., Gunter, W., Shepard, J., Foreman, R., and Myers, D. (2001). Stereotaxic delivery of corticosterone to the amygdala modulates colonic sensitivity in rats. Brain Res. 893, 135-142.

Greenwood-Van Meerveld, B., Johnson, A., Schulkin, J., and Myers, D. (2006). Long-term expression of corticotropin-releasing factor (CRF) in the paraventricular nucleus of the hypothalamus in response to an acute colonic inflammation. Brain Res. 1071, 91-96.

Gue, M. (2000). Visceral hypersensitivity in response to rectal distension in female rats: role of sexual hormones. In Neurogastroenterology from the Basics to the Clinics, H. J. Krammer and M. V. Singer, eds (Dordrecht, Kluwer), pp. 758-762.

Gue, M., Del Rio-Lacheze, C., Eutamene, H., Theodorou, V., Fioramonti, J., and Bueno, L. (1997). Stress-induced visceral hypersensitivity to rectal distension in rats: role of CRF and mast cells. Neurogastroenterol. Motil. 9, 271-279.

Gue, M., Junein, J.L., and Bueno, L. (1991). Conditioned emotional response in rats enhances colonic motility through the central release of corticotropin releasing factor. Gastroenterology 100 , 964-970.

Gue, M., Tekamp,A., Tabis, N., Junien, J.L., and Bueno, L. (1994). Cholecystokinin blockade of emotional stress- and CRF-induced colonic motor alterations in rats: role of the amygdala. Brain Res. 658, 232-238.

Gulinello, M., and Smith, S. S. (2003). Anxiogenic effects of neurosteroid exposure: sex differences and altered GABAA receptor pharmacology in adult rats. J. Pharmacol. Exp. Ther.305, 541-358.

Gunter, W. D., Shepard, J. D., Foreman, R. D., Myers, D. A., and Greenwood-Van Meerveld, B. (2000). Evidence for visceral hypersensitivity in high-anxiety rats. Physiol. Behav. 69, 379-382.

Heitkemper, M., Jarrett, M., Bond, E. F., and Chang, L. (2003). Impact of sex and gender on irritable bowel syndrome. Biol. Res. Nurs. 5, 56-65.

Heitkemper, M., Jarrett, M., Cain, K., Shaver, J., Bond, E., Woods, N. F. and Walker, E. (1996). Increased urine catecholamines and cortisol in women with irritable bowel syndrome. Am. J. Gastroenterol. 91, 906-913.

Heitkemper,M.M., Jarrett, M., Cain, K. C., Shaver, J., Walker, E., and Lewis, L. (1995). Daily gastrointestinal symptoms in women with and without a diagnosis of IBS. Dig. Dis. Sci. 40, 1511-1519.

Hermanson, O., Larhammar, D., and Blomqvist, A. (1998). Preprocholecystokinin mRNAexpressing neurons in the rat parabrachial nucleus: subnuclear localization, efferent projection, and expression of nociceptive-related intracellular signaling substances. J. Comp. Neurol. 400, 255-270.

Hertig, V. L., Cain, K. C., Jarrett, M. E., Burr, R. L., and Heitkemper, M. M. (2007). Daily stress and gastrointestinal symptoms in women with irritable bowel syndrome. Nurs. Res. 56, 399-406.

Hu, H. J., and Gereau, R. W. (2003). ERK integrates PKA and PKC signaling in superficial dorsal horn neurons. II. Modulation of neuronal excitability. J. Neurophysiol. 90, 1680-1688.

Hulisz, D. (2004). The burden of illness of irritable bowel syndrome: current challenges and hope for the future. J. Manag. Care Pharm. 10, 299-309.

Hunt, S. P., and Mantyh, P.W. (2001). The molecular dynamics of pain control. Nat. Rev. Neurosci. 2, 83-91.

Ji, G., Fu, Y., Ruppert, K. A., and Neugebauer, V. (2007). Pain-related anxiety-like behavior requires CRF1 receptors in the amygdala. Mol. Pain 3,13 .

Ji, G., and Neugebauer, V. (2008). Pro- and anti-nociceptive effects of corticotropin-releasing factor (CRF) in central amygdala neurons are mediated through different receptors. J. Neurophysiol. 99, 1201-1212.

Ji, Y., Murphy, A. Z., and Traub, R. J. (2003). Estrogen modulates the visceromotor reflex and responses of spinal dorsal horn neurons to colorectal stimulation in the rat. J. Neurosci. 23, 3908-3915.

Ji, Y., Tang, B., and Traub, R. J. (2008). The visceromotor response to colorectal distention fluctuates with the estrous cycle in rats. Neuroscience 154, 1562-1567.

Johnson, A. C., Myers, B., Zinnanti, J., Tesiram, Y., Towner, R., and Greenwood-Van Meerveld, B. (2007). Investigating central pain processing involved in anxiety-induced colonic hypersensitivity using functional magnetic resonance imaging ( $\mathrm{fMRI}$ ) in the rat. Gastroenterology 132, 4 .
Jones, M. P., Dilley, J. B., Drossman, D. and Crowell, M. D. (2006). Brain-gut connections in functional GI disorders: anatomic and physiologic relationships. Neurogastroenterol. Motil. 18, 91-103.

Jones, R., Latinovic, R., Charlton, J., and Gulliford, M. (2006). Physical and psychological co-morbidity in irritable bowel syndrome: a matched cohort study using the general practice research database. Aliment. Pharmacol. Ther. 24, 879-886.

Kayser, V., Berkley, K. J., Keita, H., Gautron, M., and Guilbaud, G. (1996). Estrous and sex variations in vocalization thresholds to hindpaw and tail pressure stimulation in the rat. Brain Res. 742, 352-354.

Krettek, J. E., and Price, J. L. (1978). Amygdaloid projections to subcortical structures within the basal forebrain and brainstem in the rat and cat. $J$. Comp. Neurol. 178, 225-254.

Lazovic, J., Wrzos, H. F., Yang, Q. X., Collins, C. M., Smith, M. B., Norgren, R., Matyas, K., and Ouyang, A. (2005). Regional activation in the rat brain during visceral stimulation detected by $c$-fos expression and fMRI. Neurogastroenterol. Motil. 17, 548-556.

LeDoux, J. E. (2000). Emotion circuits in the brain. Annu. Rev. Neurosci. 23 155-184.

LeDoux, J. E., Iwata, J., Cicchetti, P., and Reis, D. J. (1988). Different projections of the central amygdaloid nucleus mediate autonomic and behavioral correlates of conditioned fear. J. Neurosci. 8, 2517-2529.

Lisman, J., Schulman, H., and Cline, H. (2002). The molecular basis of CaMKII function in synaptic and behavioral memory. Nat. Rev. Neurosci. 3, 175-190.

Lydiard, R. B. (2001). Irritable bowel syndrome, anxiety, and depression: what are the links? J. Clin. Psychiatry $62,38-45$.

Lydiard, R. B., and Falsetti, S. A. (1999) Experience with anxiety and depression treatment studies: implications for designing irritable bowel syndrome clinical trials. Am. J. Med. 107, 65S-73S.

Lyubashina, O. A. (2004). Possible mechanisms of involvement of the amygdaloid complex in the control of gastric motor function. Neurosci. Behav. Physiol. 34, 379-388.

Marver, D. (1984). Assessment of mineralocorticoid activity in the rabbit colon. Am. J. Physiol. Renal. Physiol. 246, F437-F446.

Mawe, G. M., Coates, M. D., and Moses, P. L. (2006). Intestinal serotonin signalling in irritable bowe syndrome. Aliment. Pharmacol. Ther. 23, 1067-1076.

McDonald, A. J., Mascagni, F., and Guo, L. (1996). Projections of the medial and lateral prefrontal cortices to the amygdala: a Phaseolus vulgaris leucoagglutinin study in the rat. Neuroscience 71, 55-75.

Milner, T. A., Lubbers, L. S., Alves, S. E. and McEwen, B.S. (2008). Nuclear and extranuclear estrogen biding sites in the rat forebrain and autonomic medullary areas. Endocrinology 149, 3306-3312.

Morgan, M. A., and Pfaff, D. W. (2001). Effects of estrogen on activity and fear-related behaviors in mice. Horm. Behav. 40, 472-482.

Morsink, M. C., Steenbergen, P. J., Vos, J. B., Karst, H., Joels, M., De Kloet, E. R., and Datson, N.A. (2006). Acute activation of hippocampal glucocorticoid receptors results in different waves of gene expression throughout time. J. Neuroendocrinol. 18, 239-252.

Munck, A., and Naray-Fejes-Toth, A. (1992). The ups and downs of glucocorticoid physiology: permissive and suppressive effects revisited. Mol. Cell. Endocrinol. 90, C1-C4.

Myers, B., Dittmeyer, K., and GreenwoodVan Meerveld, B. (2007). Involvement of amygdaloid corticosterone in altered visceral and somatic sensation. Behav. Brain Res. 181, 163-167.

Myers, B., and Greenwood-Van Meerveld, B. (2007). Corticosteroid receptor-mediated mechanisms in the amygdala regulate anxiety and colonic sensitivity. Am. J. Physiol. Gastrointest. Liver Physiol. 292, G1622-G1629.

Myers, D.A., Gibson, M., Schulkin, J., and Greenwood-Van Meerveld, B. (2005). CORT implants to the amygdala and type 1 CRH receptor regulation: effect on behavior and colonic sensitivity. Behav. Brain Res. 161, 39-44.

Naliboff, B., Berman, S., Chang, L., Debbyshire, S., Suyenobu, B., and Mayer, E. (2003). Sex-related differences in IBS patients: central processing of visceral stimuli. Gastroenterology 124, 1738-1747.

Naliboff, B. D., Berman, S., Suyenobu, B. Labus, J. S., Chang, L., Stains, J., Mandelkern, M. A., and Mayer, E. A. (2006). Longitudinal change in perceptual and brain activation response to visceral stimuli in IBS patients. Gastroenterology 131, 352-365.

Naliboff, B. D., Derbyshire, S. W., Munakata, J., Berman, S., Mandelkern, M., Chang, L., and Mayer, E. A. (2001). Cerebral activation in patients with irritable bowel syndrome and control subjects during 
rectosigmoid stimulation. Psychosom. Med. 63, 365-375.

Naliboff, B. D., Waters, A. M., Labus, J. S., Kilpatrick, L., Craske, M. G., Chang, L., Negoro, H., Ibrahimovic, H., Mayer, E. A., and Ornitz, E. (2008). Increased acoustic startle responses in IBS patients during abdominal and nonabdominal threat. Psychosom. Med. 70, 920-927.

Niedringhaus, M., Jackson, P. G., Evans, S. R., Verbalis, J. G., Gillis, R. A., and Sahibzada, N. (2008). Dorsal motor nucleus of the vagus: a site for evoking simultaneous changes in crural diaphragm activity, lower esophageal sphincter pressure, and fundus tone. Am. J. Physiol. Regul. Integr. Comp. Physiol. 294, R121-R131.

Pace, T., and Spencer, R. (2005).Disruption of mineralocorticoid receptor function increases corticosterone responding to a mild, but not moderate, psychological stressor. Am. J. Physiol. Endocrinol. Metab. 288, E1082-E1088.

Pandey, S. C., Zhang, H., Roy, A., and Misra, K. (2006). Central and medial amygdaloid brain-derived neurotrophic factor signaling plays a critical role in alcohol-drinking and anxiety-like behaviors. J. Neurosci. 26, 8320-8331.

Parsons, B.,Rainbow, T.C.,MacLusky, N. J., and McEwen, B. S. (1982). Progestin receptor levels in rat hypothalamic and limbic nuclei. J. Neurosci. 2, 1446-1452.

Phelps, E. A., and LeDoux, J. E. (2005). Contributions of the amygdala to emotion processing: from animal models to human behavior. Neuron 48, 175-187.

Prewitt, C. M., and Herman, J. P. (1994). Lesion of the central nucleus of the amygdala decreases basal CRH mRNA expression and stress-induced ACTH release. Ann. N. Y. Acad. Sci. 746, 438-440.

Rainbow,T.C.,Parsons, B.,MacLusky, N. J., and McEwen, B. S. (1982). Estradiol receptor levels in rat hypothalamic and limbic nuclei. J. Neurosci. 2, 1439-1445.

Rainnie, D. G., Bergeron, R., Sajdyk, T. J., Patil,M., Gehlert,D. R., and Shekhar, A. (2004). Corticotrophin releasing factor-induced synaptic plasticity in the amygdala translates stress into emotional disorders. J. Neurosci. 24, 3471-3479.

Riedl, A., Schmidtmann, M., Stengel, A., Goebel, M., Wisser, A. S., Klapp, B. F., and Mönnikes, H. (2008). Somatic comorbidities of irritable bowel syndrome: a systematic analysis. $J$. Psychosom. Res. 64, 573-582.
Ritchie, J. (1973). Pain from distension of the pelvic colon by inflating a balloon in the irritable colon syndrome. Gut 14,125-123.

Roozendaal, B., Koolhaas, J. M., and Bohus, B. (1990). Differential effect of lesioning of the central amygdala on the bradycardiac and behavioral response of the rat in relation to conditioned social and solitary stress. Behav. Brain Res. 41, 39-48.

Rosen, J. B., and Davis, M. (1988). Enhancement of acoustic startle by electrical stimulation of the amygdala. Behav. Neurosci. 102, 195-202.

Rosztocy, A., Fioramonti, J., Jarmay, K., Barreau, F., Wittmann, T., and Bueno, L. (2003). Influence of sex and experimental protocol on the effect of maternal deprivation on rectal sensitivity to distension in the adult rat. Neurogastroenterol. Motil. 15, 679-686.

Rueter, L. E., and Jacobs, B. L. (1996). A microdialysis examination of serotonin release in the rat forebrain induced by behavioral/environmental manipulations. Brain Res. 739, 57-69.

Sah, P., Faber,E. S., Lopez De Armentia, M., and Power, J. (2003). The amygdaloid complex: anatomy and physiology. Physiol. Rev. 83 803-834.

Sakanaka, M.,Shibasaki, T., and Lederis, K. (1986). Distribution and efferent projections of corticotropin-releasing factor-like immunoreactivity in the rat amygdaloid complex. Brain Res. 382, 213-238.

Sapolsky, R. M., McEwen, B. S., and Rainbow, T. C. (1983). Quantitative autoradiography of $[3 \mathrm{H}]$ corticosterone receptors in rat brain. Brain Res. 271, 331-334.

Sapsed-Byrne, S., Ma, D., Ridout, D., and Holdcroft, A. (1996). Estrous cycle phase variations in visceromotor and cardiovascular response to colonic distension in the anesthetized rat. Brain Res. 742, 10-16.

Schulkin, J., McEwen, B. S., and Gold, P. W. (1994). Allostasis, amygdala, and anticipatory angst. Neurosci. Biobehav. Rev. 18, 389-396.

Schwaber, J. S., Kapp, B. S., Higgins, G. A., and Rapp, P. R. (1982). Amygdaloid and basal forebrain direct connections with the nucleus of the solitary tract and the dorsal motor nucleus. J. Neurosci. 2, 1424-1438.

Schwetz, I., Bradesi, S., McRoberts, J. A., Sablad, M., Miller, J. C., Zhou, H., Ohnig, G., and Mayer, E. A. (2004). Delayed stress-induced colonic hypersensitivity in male Wistar rats: role of neurokinin- 1 and corticotropinreleasing factor-1 receptors. Am. J.
Physiol. Gastrointest. Liver Physiol. 286, G683-G691.

Seeman, M. V. (1997). Psychopathology in women and men: focus on female hormones. Am. J. Psychiatry 154 1641-1647.

Shepard, J. D., Barron, K. W., and Myers, D. A. (2000). Corticosterone delivery to the amygdala increases corticotropin-releasing factor mRNA in the central amygdaloid nucleus and anxiety-like behavior. Brain Res. 861 , 288-295.

Shepard, J. D., and Myers, D. A. (2008). Strain differences in anxiety-like behavior: association with corticotropin-releasing factor. Behav. Brain Res. 186, 239-245.

Shi, C. J., and Cassell, M. D. (1998) Cortical, thalamic, and amygdaloid connections of the anterior and posterior insular cortices. J. Comp. Neurol. $399,440-468$.

Slack, S. E., Pezet, S., McMahon, S. B., Thompson, S. W., and Malcangio, M. (2004). Brain derived neurotrophic factor induces NMDA receptor subunit one phosphorylation via ERK and PKC in the rat spinal cord. Eur. J. Neurosci. 20, 1769-1778.

Stutzmann, G. E., McEwen, B. S., and LeDoux, J. E. (1998). Serotonin modulation of sensory inputs to the lateral amygdala: dependency on corticosterone. J. Neurosci. 18, 9529-9538.

Swanson, L. W. (2000). Cerebral hemisphere regulation of motivated behavior. Brain Res. 886, 113-164.

Swanson, L. W., and Petrovich, G. D. (1998). What is the amygdala? Trends Neurosci. 21,323-331.

Swiergiel, A. H., Takahashi, L. K., and Kalin, N. H. (1993). Attenuation of stress-induced behavior by antagonism of corticotrophin-releasing factor receptors in the central amygdala in the rat. Brain Res. 623, 229-234.

Tache, Y., and Bonaz, B. (2007). Corticotropin-releasing factor receptors and stress-related alterations of gut motor function. J. Clin. Invest. $117,33-40$.

Tache, Y., and Brunnhuber, S. (2008). From Hans Selye's discovery of biological stress to the identification of corticotropin-releasing factor signaling pathways: implication in stressrelated functional bowel diseases. Ann N. Y. Acad. Sci. 1148, 29-41.

Tache, Y., Monnikes, H., Bonaz, B., and Rivier, J. (1993). Role of CRF in stressrelated alterations of gastric and colonic motor function. Ann. N. Y. Acad. Sci. 697, 233-243.

Tache, Y., and Perdue, M. H. (2004) Role of peripheral CRF signaling pathways in stress-related alterations of gut motility and mucosal function. Neurogastroenterol. Motil. 1, 137-142.

Talley, N., Zinsmeister, A., Van Dyke, C., and Melton, L. (1992). Epidemiology of colonic symptoms and the irritable bowel syndrome. Gastroenterology 101, 927-934.

Tyler, K., Moriceau, S., Sullivan, R. M., and Greenwood-Van Meerveld, B. (2007). Long-term colonic hypersensitivity in adult rats induced by neonatal unpredictable vs. predictable shock. Neurogastroenterol. Motil. 19, 761-768.

Valentino, R. J., Miselis, R. R., and Pavcovich, L. A. (1999). Pontine regulation of pelvic viscera: pharmacological target for pelvic visceral dysfunctions. Trends Pharmacol. Sci. 20, 253-260.

Vamvakopoulos, N. C., and Chrousos, G. P. (1993). Evidence of direct estrogenic regulation of human corticotropin-releasing hormone gene expression. Potential implications for the sexual dimophism of the stress response and immune/inflammatory reaction. J. Clin. Invest. 92, 1896-1902.

Van Wingen, G. A., van Broekhoven, F, Verkes, R. J., Petersson, K. M. Bäckström, T., Buitelaar, J. K., and Fernández, G. (2008). Progesterone selectively increases amygdala reactivity in women. Mol. Psychiatry 13, 325-333.

Verne, G. N., Robinson, M. E., and Price, D. D. (2001). Hypersensitivity to visceral and cutaneous pain in the irritable bowel syndrome. Pain 2001, 93, 7-14.

Viau, V., and Meaney, M. J. (1991). Variations in the hypothalamicpituitary-adrenal response to stress during the estrous cycle in the rat. Endocrinology 129, 2503-2511.

Wang, Z., Bradesi, S., Maarek, J.M., Lee, K., Winchester, W. J., Mayer, E. A., and Holschneider, D. P. (2008). Regional brain activation in conscious, nonrestrained rats in response to noxious visceral stimulation. Pain 138 233-243.

Weiser, M. J., and Handa, R. J. (2009). Estrogen impairs glucocorticoid dependent negative feedback on the hypothalamic-pituitary-adrenal axis via estrogen receptor alpha within the hypothalamus. Neuroscience 159, 883-895.

Weiskrantz, L. (1956). Behavioral changes associated with ablation of the amygdaloid complex in monkeys. J. Comp. Physiol. Psychol. 49, 381-391.

Whitehead, W. E., Crowell, M. D., Robinson, J. C., Heller, B. R., and Schuster, M. M. (1992). Effects of 
stressful life events on the bowel symptoms: subjects with irritable bowel syndrome compared with subjects without irritable bowel dysfunction. Gut 30, 825-830.

Whitehead, W. E., Engel, B. T., and Schuster, M. M. (1980). Irritable bowel syndrome: physiological and psychological differences between diarrhea-predominant and constipation-predominant patients. Dig. Dis. Sci. 25, 404-413.

Whitehead, W. E., Holtkotter, B., Enck, P., Hoelzl, R., Holmes, K. D., Anthony, J., Shabsin, H. S., and
Schuster, M.M. (1990). Tolerance for rectosigmoid distention in irritable bowel syndrome. Gastroenterology 98 , 1187-1192.

Whitehead, W. E., Palsson, O., and Jones, K. R. (2002). Systematic review of the comorbidity of irritable bowel syndrome with other disorders: what are the causes and implications? Gastroenterology 122, 1140-1156.

Wilder-Smith, C., Schindler, D., Lovblad, K., Redmond, S., and Nirkko, A. (2004). Brain functional magnetic resonance imaging of rectal pain and activation of endogenous inhibitory mechanisms in irritable bowel syndrome patient subgroups and healthy controls. Gut 53, 1595-1601.

Conflict of Interest Statement: The authors have no conflict of interest to declare related to this work.

Received: 08 April 2009; paper pending published: 27 April 2009; accepted: 27 May 2009; published online: 10 June 2009.

Citation: Myers $B$ and GreenwoodVan Meerveld B (2009) Role of anxiety in the pathophysiology of irritable bowel syndrome: importance of the amygdala. Front. Neurosci. 3:47. doi: 10.3389/neuro.21.002.2009

This article was submitted to Frontiers in Autonomic Neuroscience, a specialty of Frontiers in Neuroscience.

Copyright $(02009$ Myers and GreenwoodVan Meerveld. This is an open-access article subject to a non-exclusive license between the authors and Frontiers Media SA, which permits use, distribution and reproduction in other forums, provided the original authors and source are credited and other Frontiers conditions are complied with. 